\title{
SÍNTESE E CARACTERIZAÇÃO DE CATALISADORES V/Cr SUPORTADO EM Nb/AI E AVALIAÇÃO CATALÍTICA NA DECOMPOSIÇÃO DO ISOPROPANOL
}

\author{
M. C. de ALMEIDA MONTEIRO ${ }^{1}$, J. A. J. RODRIGUES ${ }^{2}$, G. G. CORTEZ ${ }^{1}$
}

${ }^{1}$ Departamento de Engenharia Química, Laboratório de Catálise I - EEL-USP, Lorena-SP,

2 Instituto Nacional de Pesquisas Espaciais - LCP

E-mail para contato: maria@alunos.eel.usp.br

\begin{abstract}
RESUMO - Aluminas de transição são materiais importantes em inúmeras reações catalíticas, podendo atuar como catalisador ou como suporte catalítico para metais. A reação de decomposição do isopropanol permitiu observar que a adição de vanádio e cromo no suporte alumina-nióbio modificou a atividade catalítica devido à diminuição de sítios ácidos sobre o suporte.
\end{abstract}

\section{INTRODUÇÃO}

A fase de transição da alumina mais utilizada em catálise é a $\gamma-\mathrm{Al}_{2} \mathrm{O}_{3}$, devido principalmente a sua elevada área superficial se comparada às outras fases de aluminas (Baumann et al., 2005).

Óxidos de nióbio são considerados catalisadores seletivos para a desidrogenação oxidativa $(\mathrm{ODH})$ do propano, porém a promoção de óxido de nióbio com vanádio promove um aumento da atividade catalítica, sem comprometer a seletividade do propano à olefina (Ross et al., 1993). O desempenho catalítico de catalisadores de $\mathrm{Cr}_{2} \mathrm{O}_{3}$ suportados foi discutido no trabalho de Al-Zahrani et al. (2001), mostrando que o efeito promotor do cromo sobre o desempenho do catalisador poderia estar relacionado à resistência da ligação entre o oxigênio e a estrutura do suporte do catalisador.

O objetivo deste trabalho foi caracterizar e avaliar as propriedades ácidas e/ou básicas de catalisadores $\mathrm{V} / \mathrm{Cr}$ suportados sobre $\mathrm{Nb} / \mathrm{Al}$ na reação decomposição do isopropanol.

\section{METODOLOGIA}

Sintetizou-se uma pseudoboehmita pelo preparo de uma solução aquosa de aluminato de sódio e solução de sulfato de alumínio. Adicionou-se o agente precipitante $\mathrm{NaAlO}_{2}$ à solução de $\mathrm{Al}_{2}\left(\mathrm{SO}_{4}\right)_{3}$, com temperatura em $60^{\circ} \mathrm{C}$. Em seguida realizou-se a lavagem do precursor, a amostra foi seca em estufa a $50^{\circ} \mathrm{C}$ e calcinada até $500^{\circ} \mathrm{C}$. A alumina foi impregnada com solução aquosa de $\mathrm{NH}_{4}\left[\mathrm{NbO}\left(\mathrm{C}_{2} \mathrm{O}_{4}\right)_{2}\left(\mathrm{H}_{2} \mathrm{O}\right)_{2}\right]\left(\mathrm{H}_{2} \mathrm{O}\right)_{n}$ aquecida a $70^{\circ} \mathrm{C}$, de modo que a porcentagem de óxido de nióbio $\left(\mathrm{Nb}_{2} \mathrm{O}_{5}\right)$ na alumina $\left(\mathrm{Al}_{2} \mathrm{O}_{3}\right)$ fosse $5 \%(\mathrm{p} / \mathrm{p})$. Adicionou-se a alumina ao balão do rotoevaporador e o solvente foi evaporado sob vácuo. $\mathrm{O}$ pó residual foi seco a $110^{\circ} \mathrm{C}$ em estufa, e calcinado a $600^{\circ} \mathrm{C}$ em mufla, obtendo-se assim o suporte $\mathrm{Al}_{2} \mathrm{O}_{3} / \mathrm{Nb}_{2} \mathrm{O}_{5}$. Na preparação dos catalisadores, utilizou-se uma solução aquosa de $\mathrm{Cr}\left(\mathrm{NO}_{3}\right)_{3} \cdot 9 \mathrm{H}_{2} \mathrm{O}$ e a impregnação foi realizada pelo mesmo método descrito acima. $\mathrm{O}$ pó 
residual foi seco a $110^{\circ} \mathrm{C}$ em estufa, e depois calcinado a $450^{\circ} \mathrm{C}$, obtendo-se assim o catalisador identificado por $4 \mathrm{Cr} / \mathrm{NbAl}$. Utilizando-se soluções aquosas de $\mathrm{Cr}\left(\mathrm{NO}_{3}\right)_{3} .9 \mathrm{H}_{2} \mathrm{O}$ e $\mathrm{NH}_{4} \mathrm{VO}_{3}$, através do método de co-impregnação, obteve-se os catalisadores identificados por $\mathrm{VCr} / \mathrm{NbAl}$. No catalisador impregnado apenas com cromo, utilizou-se 4 átomos de cromo por $\mathrm{nm}^{2}$ de suporte. No catalisador co-impregnado a quantidade total de cromo e vanádio foi de 8 átomo por $\mathrm{nm}^{2}$ de alumina, um com 4 átomos de cromo e 4 átomos de vanádio, e outro com 6 átomos de vanádio e 2 átomos de cromo.

\section{RESULTADOS E DISCUSSÕES}

A área superficial $\left(\mathrm{S}_{\mathrm{BET}}\right)$, o volume de poros $\left(\mathrm{V}_{\mathrm{p}}\right)$ e o diâmetro médio de poros $\left(\mathrm{D}_{\mathrm{p}}\right)$ das amostras calcinadas são apresentados na Tabela 1.

Tabela 1 - Valores de área específica, volume de poros e diâmetro de poros do suporte e catalisadores.

\begin{tabular}{|c|c|c|c|c|c|c|}
\hline $\begin{array}{c}\text { Suporte/ } \\
\text { catalisadores }\end{array}$ & $\begin{array}{c}\mathrm{Nb}_{2} \mathrm{O}_{5} \\
(\% \mathrm{p} / \mathrm{p})\end{array}$ & $\begin{array}{c}\mathrm{V}_{2} \mathrm{O}_{5} \\
(\% \mathrm{p} / \mathrm{p})\end{array}$ & $\begin{array}{c}\mathrm{Cr}_{2} \mathrm{O}_{3} \\
(\% \mathrm{p} / \mathrm{p})\end{array}$ & $\begin{array}{c}\mathrm{S}_{\mathrm{BET}} \\
\left(\mathrm{m}^{2} \cdot \mathrm{g}^{-1}\right)\end{array}$ & $\begin{array}{c}\mathrm{V}_{\mathrm{p}} \\
\left(\mathrm{cm}^{3} \cdot \mathrm{g}^{-1}\right)\end{array}$ & $\begin{array}{c}\mathrm{D}_{\mathrm{p}} \\
(\AA)\end{array}$ \\
\hline $\mathrm{Al}_{2} \mathrm{O}_{3}$ & 0 & 0 & 0 & 366 & 1,68 & 160 \\
\hline $\mathrm{Nb} / \mathrm{Al}$ & 5 & 0 & 0 & 300 & 0,59 & 99 \\
\hline $4 \mathrm{Cr} / \mathrm{NbAl}$ & 5 & 0 & 23,2 & 277 & 0,40 & 47 \\
\hline $4 \mathrm{~V} / 4 \mathrm{Cr} / \mathrm{NbAl}$ & 5 & 26,6 & 23,2 & 236 & 0,43 & 38 \\
\hline $6 \mathrm{~V} / 2 \mathrm{Cr} / \mathrm{NbAl}$ & 5 & 35,1 & 13,2 & 230 & 0,45 & 82 \\
\hline
\end{tabular}

Os resultados mostraram que a área específica, o volume de poros e o diâmetro médio de poros da alumina diminuíram com a impregnação do nióbio. A adição de cromo e a coimpregnação com vanádio e cromo sobre o suporte $\mathrm{Nb} / \mathrm{Al}$ provocaram uma diminuição desses três parâmetros, porém a diminuição da área específica foi maior nos dois catalisadores com cromo e vanádio, se comparado ao catalisador apenas com cromo, enquanto o diâmetro de poros diminuiu mais com a adição de quantidades iguais de vanádio e cromo sobre o suporte.

As Figuras 1 e 2 apresentam as isotermas adsorção-dessorção de $\mathrm{N}_{2}$ e distribuição de volume de poros, respectivamente, da $\gamma$-alumina, do suporte e dos catalisadores. Pela Figura 1 , percebe-se que tanto a $\gamma$-alumina como o suporte e os catalisadores se enquadram na isoterma do tipo IV, característica de materiais mesoporosos (Donohue e Aranovich, 1999). A Figura 2 permite observar que a $\gamma$-alumina apresenta um comportamento monomodal, com distribuição de poros na faixa de 10 a $60 \AA$ (microporos). O suporte $\mathrm{Nb} / \mathrm{Al}$ apresenta maior quantidade de poros na faixa de 60 a $160 \AA$, indicando que o nióbio adicionado se depositou nos poros de menor diâmetro. O catalisador $4 \mathrm{Cr} / \mathrm{NbAl}$ apresenta em sua maioria poros de $20 \mathrm{a}$ $60 \AA ̊$ e de 80 a $160 \AA$, que pode ser observada pela diminuição do diâmetro de poros, em relação ao suporte, constatada pela análise utilizando o método BET. A co-impregnação de vanádio e cromo ao suporte provocou uma diminuição do número de poros com 20 a $60 \AA$ e aumentou o número de poros com diâmetro entre 80 e $160 \AA$, mostrando que o vanádio adicionado se depositou nos poros de menor diâmetro.

Figura 1 - Isotermas de $\mathrm{N}_{2}$ da $\gamma$-alumina, do suporte e dos catalisadores. 

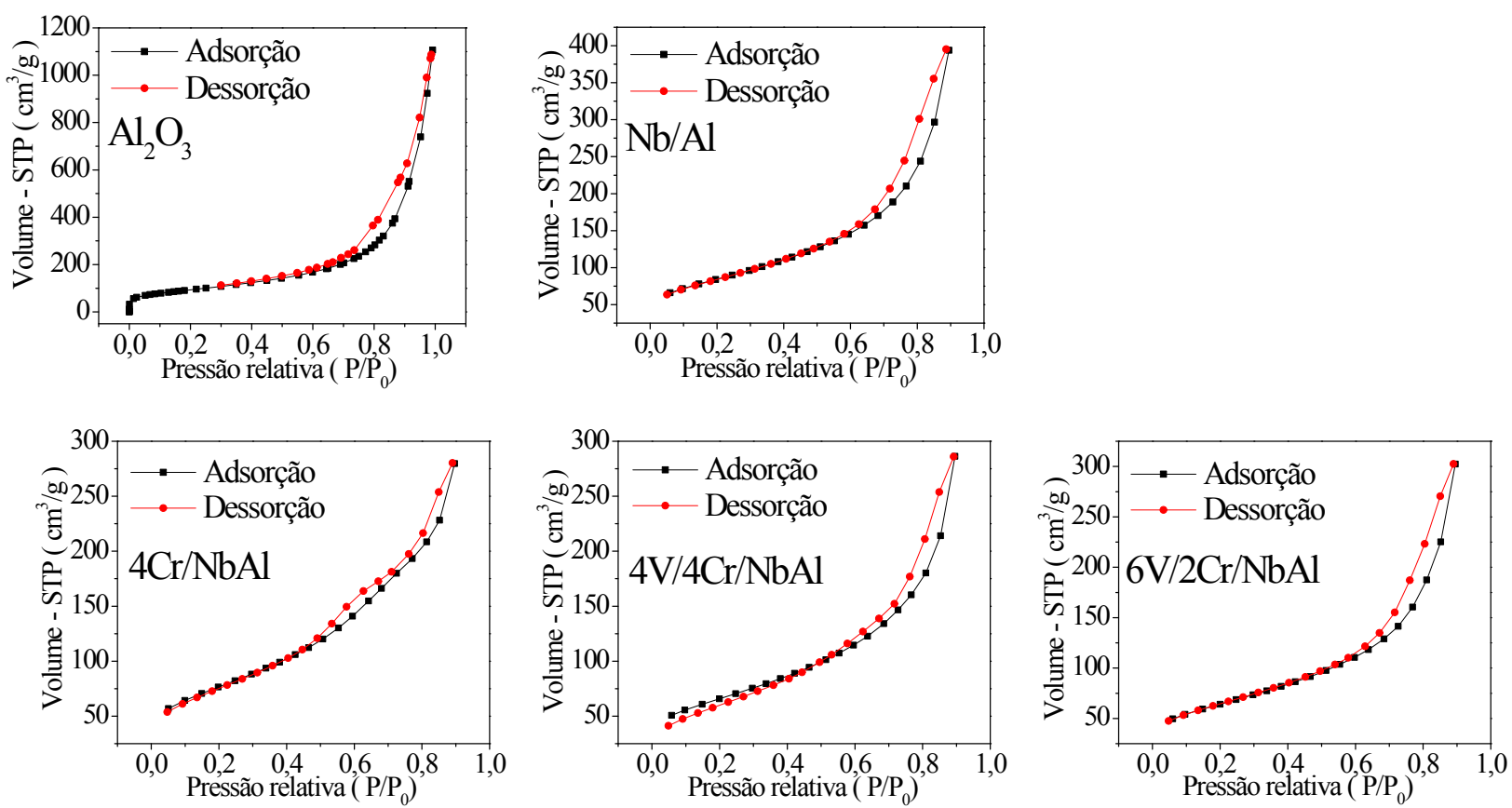

Figura 2 - Distribuição de volume de poros por diâmetro da $\gamma$-alumina, do suporte e dos catalisadores.
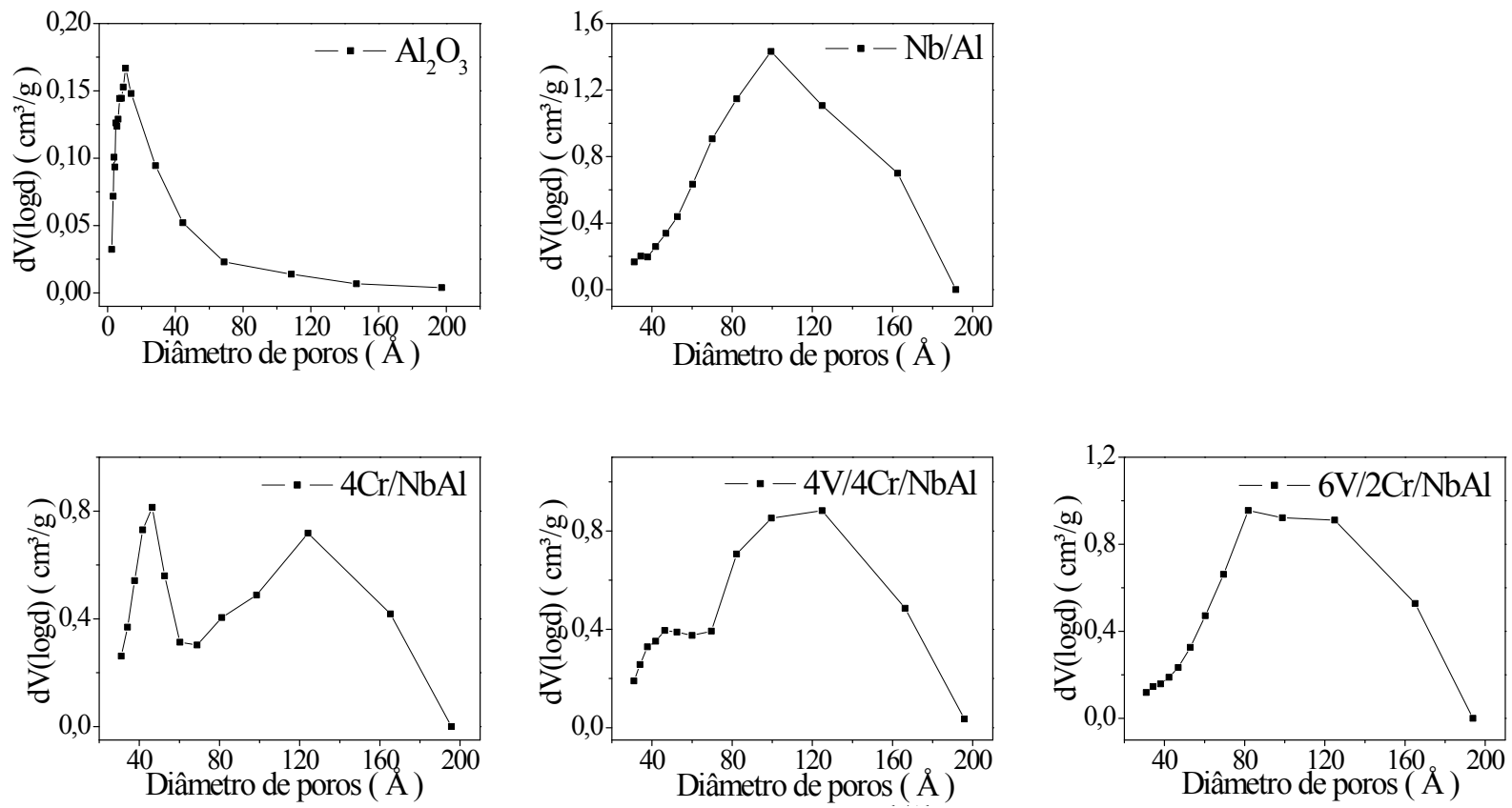

A Figura 3 representa o difratograma de raios $X$ do precursor, da $\gamma$-alumina, do suporte e dos catalisadores.

Figura 3 - Difratograma de raios $\mathrm{X}$ do precursor, da $\gamma$-alumina e do suporte e catalisadores. Picos referentes às fases $(\bullet)$ boehmita, $(\square) \gamma-\mathrm{Al}_{2} \mathrm{O}_{3}$ e $(\square) \mathrm{Cr}_{2} \mathrm{O}_{3}$. 


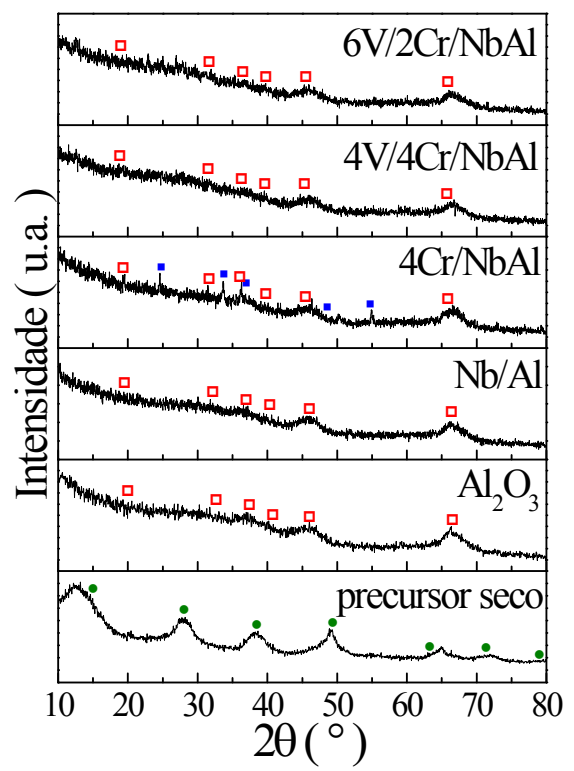

Os resultados de DRX mostraram que o precursor é constituído por boehmita, e, portanto, o suporte e os catalisadores são constituídos por $\gamma$-alumina. A adição de cromo ao suporte ocasionou o aparecimento de picos mais intensos em $2 \theta$ iguais a $33,56^{\circ}$ e $36,19^{\circ}$ e um terceiro pico em $2 \theta$ igual a 54,65 (Ma et al., 2011), referentes ao $\mathrm{Cr}_{2} \mathrm{O}_{3}$, e a co-impregnação com vanádio e cromo não modificou o difratograma de raios $\mathrm{X}$ do suporte, porque os seus óxidos podem estar presentes em um estado amorfo ou encontram-se altamente dispersos sobre o suporte.

Os perfis de redução do $\mathrm{V}_{2} \mathrm{O}_{5}$ e $\mathrm{Cr}_{2} \mathrm{O}_{3}$ mássicos e do suporte e catalisadores são mostrados na Figura 4. O óxido de vanádio mássico apresenta três temperaturas máximas de redução a 655, 690 e $823{ }^{\circ} \mathrm{C}$, que corresponde a seguinte etapa de redução das espécies de vanádio, descrito por Korane et al. (1994):

$$
\mathrm{V}_{2} \mathrm{O}_{5} \rightarrow \mathrm{V}_{6} \mathrm{O}_{13}\left(675^{\circ} \mathrm{C}\right) ; \mathrm{V}_{6} \mathrm{O}_{13} \rightarrow \mathrm{V}_{2} \mathrm{O}_{4}\left(705^{\circ} \mathrm{C}\right) ; \mathrm{V}_{2} \mathrm{O}_{4} \rightarrow \mathrm{V}_{2} \mathrm{O}_{3}\left(780^{\circ} \mathrm{C}\right)
$$

$\mathrm{O}$ perfil de RTP do $\mathrm{Cr}_{2} \mathrm{O}_{3}$ mássico indica um pico de redução a $421^{\circ} \mathrm{C}$. De acordo com Ma et al. (2011), os perfis de redução apresentam um pico entre 250 e $450^{\circ} \mathrm{C}$, que é atribuído à redução das espécies $\mathrm{Cr}^{6+}$ à estrutura $\mathrm{Cr}_{2} \mathrm{O}_{3}\left(\mathrm{Cr}^{3+}\right)$. Pela Figura 4, percebe-se que a redução a temperatura programada da amostra $4 \mathrm{Cr} / \mathrm{NbAl}$ mostrou um pico de redução a $373^{\circ} \mathrm{C}$ devido a redução do $\mathrm{Cr}^{6+} \mathrm{a} \mathrm{Cr}^{3+}$. Houve o aparecimento de um pico de redução do vanádio à $529^{\circ} \mathrm{C} \mathrm{e}$ $583^{\circ} \mathrm{C}$, respectivamente, nas amostras $4 \mathrm{~V} / 4 \mathrm{Cr} / \mathrm{NbAl}$ e $6 \mathrm{~V} / 2 \mathrm{Cr} / \mathrm{NbAl}$, correspondente à redução de $\mathrm{V}^{5+} \mathrm{a} \mathrm{V}^{4+}$, mostrando que o pico de redução pode se devido à interação do vanádio com o suporte. A análise da amostra $4 \mathrm{~V} / 4 \mathrm{Cr} / \mathrm{NbAl}$ mostrou um pico de redução do cromo a $374^{\circ} \mathrm{C}$, enquanto a amostra $6 \mathrm{~V} / 2 \mathrm{Cr} / \mathrm{NbAl}$ não apresentou pico correspondente a esse óxido, indicando que o óxido de cromo estava bem distribuído na amostra. Não houve pico de redução de nióbio em nenhuma das amostras devido à baixa concentração de $\mathrm{Nb}_{2} \mathrm{O}_{5}$ na alumina.

Figura 4 - Perfis de RTP do $\mathrm{V}_{2} \mathrm{O}_{5}$ e $\mathrm{Cr}_{2} \mathrm{O}_{3}$ mássicos e do suporte e catalisadores. 

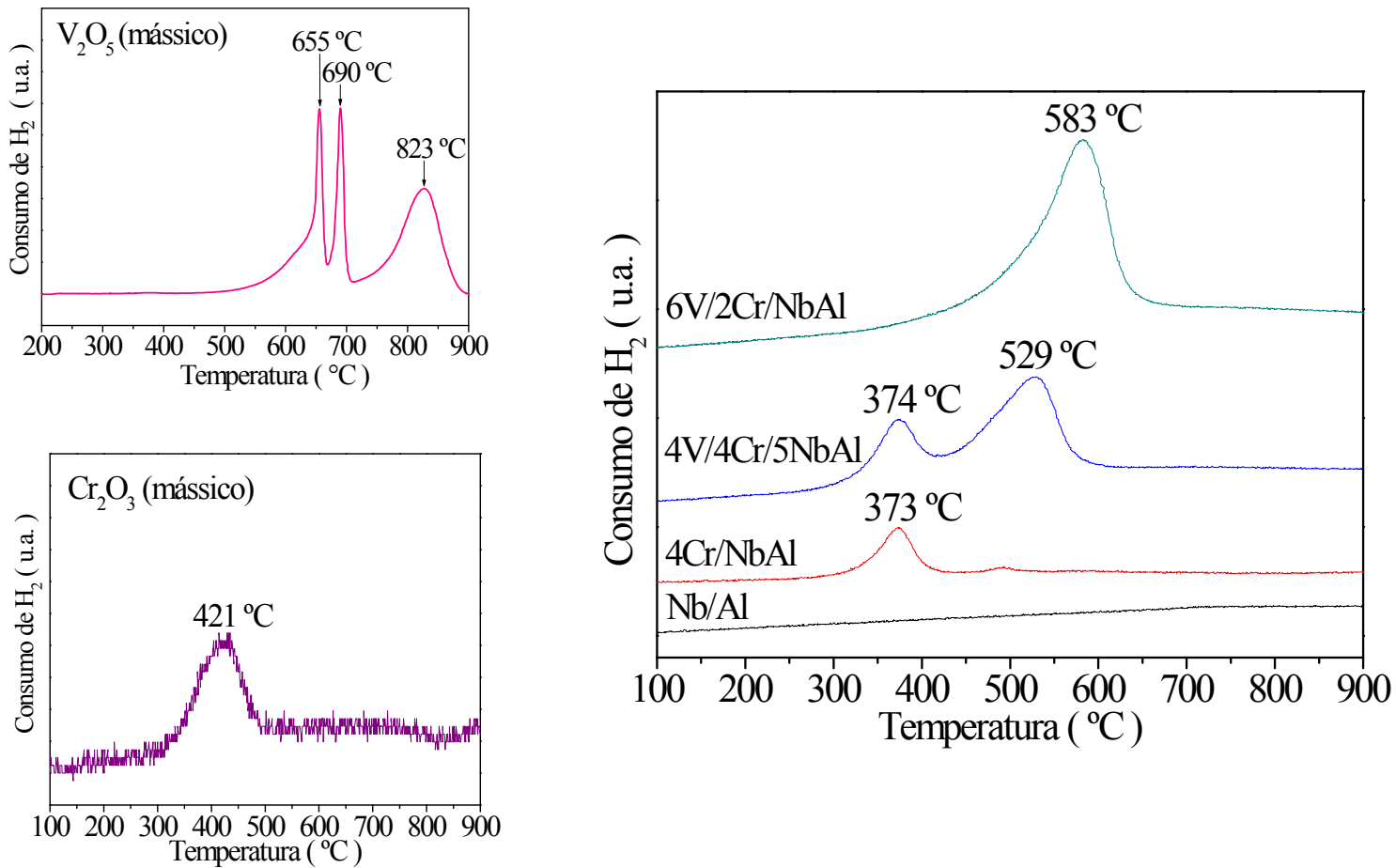

Temperatura $\left({ }^{\circ} \mathrm{C}\right)$

Tabela 2 - Resultados da taxa específica de reação (TER) e da taxa específica de formação dos produtos (TEP) dos catalisadores a $270{ }^{\circ} \mathrm{C}$.

\begin{tabular}{|c|c|c|c|}
\hline \multirow{2}{*}{ Suporte/catalisadores } & $\begin{array}{c}\text { TER } \\
\left(\mu \mathrm{mol} \cdot \mathrm{m}^{-2} \cdot \mathrm{min}^{-1}\right)\end{array}$ & \multicolumn{2}{|c|}{ TEP $\left(\mu \mathrm{mol}^{-2} \mathrm{~min}^{-1}\right) / 270^{\circ} \mathrm{C}$} \\
\cline { 3 - 4 } & 12,8 & Propeno & Éter diisopropílico \\
\hline $\mathrm{Al}_{2} \mathrm{O}_{3}$ & 24,1 & 13,2 & 0,4 \\
\hline $\mathrm{Nb} / \mathrm{Al}$ & 6,7 & 24,1 & 0,1 \\
\hline $4 \mathrm{Cr} / \mathrm{NbAl}$ & 12,0 & 6,0 & 0,4 \\
\hline $4 \mathrm{~V} / 4 \mathrm{Cr} / \mathrm{NbAl}$ & 19,7 & 12,0 & 0,0 \\
\hline $6 \mathrm{~V} / 2 \mathrm{Cr} / \mathrm{NbAl}$ & \multicolumn{2}{|c}{} \\
\hline
\end{tabular}

Os resultados da atividade catalítica obtidos na reação de decomposição do isopropanol, na temperatura de $270^{\circ}$ para uma conversão de $15 \%$ de isopropanol, são mostrados na Tabela 2. A impregnação da $\gamma$-alumina com nióbio aumentou a taxa de reação e a produção de propeno, porém diminuiu a produção de éter diisopropílico. A impregnação de cromo sobre o suporte diminuiu taxa de reação e a formação de propeno, no entanto aumentou a produção de éter diisopropílico, e a co-impregnação de vanádio e cromo aumentou a taxa de reação e a formação de propeno. $\mathrm{O}$ aumento da taxa de reação foi maior para uma maior quantidade de vanádio adicionada. A produção de éter foi nula na impregnação com quantidades iguais de vanádio e cromo, e aumentou levemente para o catalisador com maior concentração de vanádio. A diminuição da atividade catalítica após a impregnação com cromo e a coimpregnação com vanádio e cromo se deve à diminuição da acidez do suporte na presença dos metais, havendo um bloqueio sobre os sítios ácidos.

\section{CONCLUSÕES}


As propriedades texturais da $\gamma-\mathrm{Al}_{2} \mathrm{O}_{3}$ foram modificadas pela adição de nióbio, vanádio e cromo, devido ao bloqueio dos micro e mesoporos. Uma vez que o precursor apresentou a fase boehmita, o DRX das amostras calcinadas comprovou a existência da alumina de transição $\gamma-\mathrm{Al}_{2} \mathrm{O}_{3}$. Apenas o catalisador impregnado somente com cromo apresentou picos referentes a esse óxido. Nos resultados de RTP, os catalisadores apresentaram picos referentes a seus óxidos, exceto o catalisador $6 \mathrm{~V} / 2 \mathrm{Cr} / \mathrm{NbAl}$, que apresentou apenas um pico referente à redução do vanádio. A partir da reação de decomposição do isopropanol, conclui-se que os catalisadores apresentaram basicamente sítios ácidos, evidenciado pela formação de propeno e éter diisopropílico. Além disso, verificou-se que a adição apenas de cromo promoveu maior diminuição da atividade catalítica do que a co-impregnação com os dois metais.

\section{AGRADECIMENTOS}

Ao LCP-INPE de Cachoeira Paulista/SP e à FAPESP (2014/13765-8).

\section{REFERENCIAS}

Al-ZAHRANI, S. M.; ELBASHIR, N. O.; ALBASAEED, A. E.; ABDULWAHED, M., Catalytic performance of chromium oxide supported on $\mathrm{Al}_{2} \mathrm{O}_{3}$ in oxidative dehydrogenation of isobutane to isobutene. Ind. Eng. Chem. Res., v. 40, p. 781-784, 2001 .

BAUMANN, T. F.; CHINN, S. C.; SAWVEL, A. M.; MAXWELL, R. S.; SATCHER, J. H., Synthesis of high-surface-area alumina aerogels without the use of alkoxide precursors. Chem. Mat., v. 172, p. 395-401, 2005.

DONOHUE, M. D.; ARANOVICH, G. L., A new classification of isotherms for Gibbs adsorption of gases on solids. Fluid Phase Equilib., v. 158-160, p. 557-563, 1999.

KORANNE, M. M.; GOODWIN, J.G.; MARCELIN, G., Characterization of Silica- and Alumina-Supported Vanadia Catalysts Using Temperature Programmed Reduction. $J$. Catal., v. 148, p. 369-377, 1994.

ROSS, J. R. H.; SMITS, R. H. H.; SESHAN, K., The use of niobia in oxidation catalysis. Catal. Today, v. 16, p. 503-511, 1993.

MA, R; HU, P.; JIN, L.; WANG, Y.; LU, J.; LUO, M, Characterization of $\mathrm{CrO}_{\mathrm{x}} / \mathrm{Al} 2 \mathrm{O} 3$ catalysts for dichloromethane oxidation. Catal. Today, v. 175, p. 598-602, 2011. 